\title{
Applications of 2-D Moiré Deflectometry to Atmospheric Turbulence
}

\author{
S. Rasouli ${ }^{1,2 \dagger}$, M. D. Niry ${ }^{1}$, Y. Rajabi ${ }^{1}$, A. A. Panahi ${ }^{1}$ and J. J. Niemela ${ }^{3}$ \\ ${ }^{1}$ Department of Physics, Institute for Advanced Studies in Basic Sciences (IASBS), Zanjan 45137-66731, Iran \\ ${ }^{2}$ Optics Research Center, Institute for Advanced Studies in Basic Sciences (IASBS), Zanjan 45137-66731, \\ Iran \\ ${ }^{3}$ The Abdus Salam ICTP, Strada Costiera 11, 34151 Trieste, Italy \\ †Corresponding Author Email: rasouli@iasbs.ac.ir
}

(Received August 23, 2013; accepted December 1, 2013)

\begin{abstract}
We report on applications of a moiré deflectometry to observations of anisotropy in the statistical properties of atmospheric turbulence. Specifically, combining the use of a telescope with moiré deflectometry allows enhanced sensitivity to fluctuations in the wave-front phase, which reflect fluctuations in the fluid density. Such phase fluctuations in the aperture of the telescope are imaged on the first grating of the moiré deflectometer, giving high spatial resolution. In particular, we have measured the covariance of the angle of arrival (AA) between pairs of points displaced spatially on the telescope aperture which allows a quantitative measure of anisotropy in the atmospheric surface layer. Importantly, the telescope-based moiré deflectometry measures directly in the spatial domain and, besides being a non-intrusive method for studying turbulent flows, has the advantage of being relatively simple and inexpensive.
\end{abstract}

Keywords: Boundary layers: turbulence; Electromagnetic waves: atmospheric propagation; Turbulence: atmospheric; Diffraction gratings: optical; Interferometers.

\section{NOMENCLATURE}

$\begin{array}{ll}A A, \alpha & \text { angle of arrival } \\ B_{\alpha} & \text { covariance of the AA fluctuations } \\ D \varphi & \text { second order phase structure function } \\ d & \text { gratings' period } \\ \mathrm{d}_{\mathrm{m}} & \text { moiré fringe spacing } \\ f & \text { telescope focal length } \\ f^{\prime} & \text { focal length of the collimating lens } \\ \mathrm{r} & \text { distance between two points } \\ \mathrm{r}_{0} & \text { Fried parameter } \\ \mathrm{Z}_{\mathrm{k}} & \text { Talbot length } \\ \alpha_{x} & \text { component of angle of arrival in x direction }\end{array}$

\section{INTRODUCTION}

In recent years, a number of authors have measured the statistics of the wave-front phase across a telescope aperture when the telescope is collecting light through a turbulent atmosphere; in particular, a variety of sensing methods have been used to characterize both spatially and temporally the statistics of the wave-front phase. These previous works have used single (Acton et al. 1992), double (Fried 1975; Sarazin and Roddier 1990), and multiple aperture sensors (Shack-Hartmann) (Dayton et al. 1992; Nicholls et al. 1995; Silbaugh $\beta \quad$ exponent of power spectrum

$\beta_{k o l}$ exponent of Kolmogorov power spectrum

$\delta \mathrm{d}$ displacement of the gratings in a direction normal to its rulings

$\delta \mathrm{d}_{\mathrm{m}}$ moiré fringe shift

$\kappa \quad$ Gladstone-Dale constant

$\lambda$ wavelength of the incident light

$\rho$ density

$\varphi \quad$ wave-front's phase

et al. 1996) to measure the wave-front tilt. In these works, as in that of Arad et al. (1998), deviations from isotropy and homogeneity of the turbulence were described.

Optical methods, in general, can be well-suited to measurements directly in the spatial domain and have already been used to study transverse velocity structure functions, including Raman-Excited Laser Induced Electronic Fluorescence (RELIEF) (Frisch 1995; Noullez et al. 1997; Miles et al. 1991; Webster 2009). Methods such as tracking inertial particles using a high-speed camera (Volk et al. 2008) or by using the Laser Doppler effect (Volk et al. 2008) and multiple probes (Lekakis 1996; 
Hutchins et al. 2009) can be used for measurement of spatial increments of the velocity. Measurements in the temporal domain (Gagne et al. 1990; Castaing et al. 1990; Maurer et al. 1994) have found exponents of the velocity structure function, after recasting into the spatial domain using the Taylor hypothesis (Frisch 1995).

Recently, we have suggested a method with high sensitivity and resolution for probing atmospheric turbulence, based on moiré deflectometry coupled with a telescope (Rasouli 2010). Because it accesses large volumes of 2D data across the telescope aperture, this method is well-suited for studying the spatial properties of wave-fronts. Indeed, due to the magnification of the telescope, the use of the moire technique, and the Talbot effect (Patorski 1993), measurements of fluctuations in the angle of arrival (AA) can be up to 2 orders of magnitude more precise than other methods (Rasouli 2010; Rasouli and Tavassoly 2006; Rasouli and Tavassoly 2008).

We have used this method in the present work to measure statistical properties of atmospheric turbulence in both longitudinal and transverse directions with respect to the ground surface. Specifically, we calculated the covariance of AA between pairs of points displaced in space on the telescope aperture plane with a spatial resolution of $0: 35 \mathrm{~mm}$; this can be extended to calculate the exponent of the AA structure functions of arbitrary order in the spatial domain. While is expected that the AA structure function and velocity structure function are related to each other, that relationship can be complex in non-isothermal flows, and especially where temperature can play both an active and passive role. While the present observations demonstrate the potential of the method in turbulence research, it is clear that the next step will be to applications in controlled, laboratory experiments where effects can be isolated. In the field a further improvement would be to have separate, simultaneous and precise measurements of the temperature field alone, drawing from the laboratory experience effective methods of deconvolution.

\section{BASIC DEFINITIONS}

\section{Moiré Technique}

Moiré patterns appear when two similar gratings are superimposed at a small angle or when they have slightly different periods (Patorski 1993) (see Fig. $1)$.

In the moiré technique displacing one of the gratings by $\delta \mathrm{d}_{\mathrm{m}}$ in a direction normal to its rulings leads to a moiré fringe shift given by $\delta \mathrm{d}_{\mathrm{m}}=\left(\mathrm{d}_{\mathrm{m}} / \mathrm{d}\right) \delta \mathrm{d}$, where $d$ and $d_{m}$ are the period of the superimposed gratings and the moiré fringe spacing, respectively (Patorski 1993) (see the background movie of Fig. $1)$.

In many applications of the moiré pattern, one of the superimposed gratings is the image of a physical grating or is one of the self-images of the first grating (Rasouli 2012).

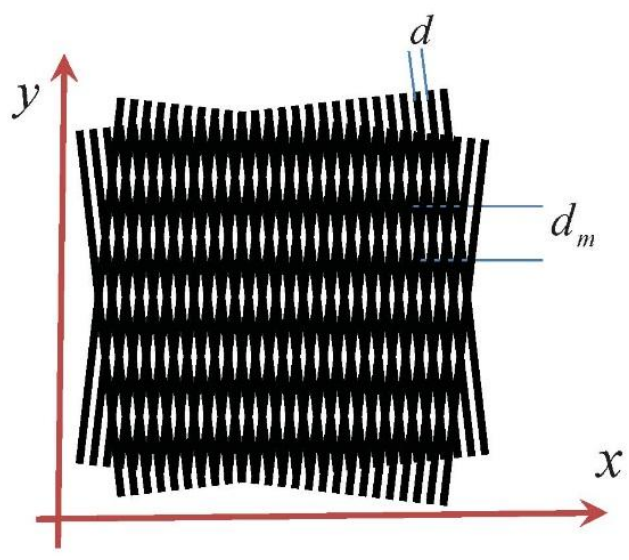

Fig. 1. (Color online) A moiré pattern, formed by superimposing two sets of parallel lines, one set rotated by an angle $\theta$ with respect to the other.

To see animation, click on panel (Movie).

\section{Talbot Effect}

By illuminating a grating or other periodic structure with a spatially coherent light beam, exact images and many other images of the structure can be found at finite distances from the grating. This selfimaging phenomenon is called the Talbot effect (Talbot 1836).

The regular distance between the grating and the imaging plane is called the Talbot length (Rayleigh 1881), $Z_{k=1}=2 d^{2} / \lambda$, where $d$ and $\lambda$ are the period of the grating and the wavelength of the incident light, respectively. The effect has found interesting applications in image processing and synthesis, photolithography, optical testing, optical metrology, and spectrometry (Patorski 1989).

\section{Moiré Deflectometry}

A moiré deflectometer consists of two gratings, in which the second grating is installed at the plane of one of the self-images of the first grating and the object under inspection is placed in front the first grating. The moiré deflectometry measures ray deflections in the paraxial approximation induced by the phase object. The resulting moiré fringe pattern is a map of ray deflections corresponding to the optical properties of the inspected object. Generally, when the image-forming light propagates in a perturbed medium the self-image is distorted and the distortion is magnified by the moiré pattern (Keren and Kafri 1985). A schematic set-up of a moiré deflectometry is shown in Fig. 2. (Also, see the background movie of Fig. 1).

\section{Angle of Arrival (AA), $\alpha$}

A measure of the propagation direction of an optical wave upon arrival at a receiver is called the Angle of Arrival (AA). AA is the angle between the actual 
plane of the phase front at the receiver and a plane of reference (Roddier 1999) (See Fig. 3).

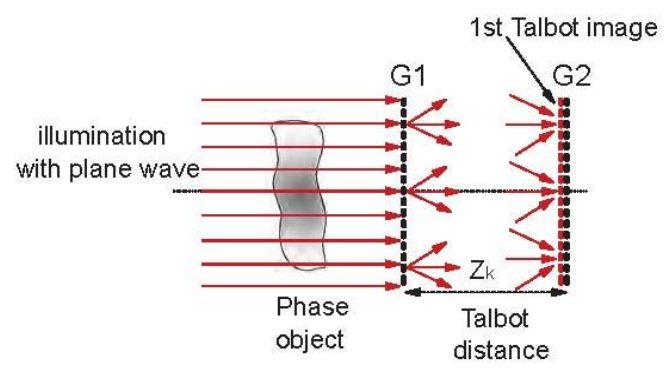

Fig. 2. (Color online) Schematic set-up of a moiré deflectometry for the study of phase objects.

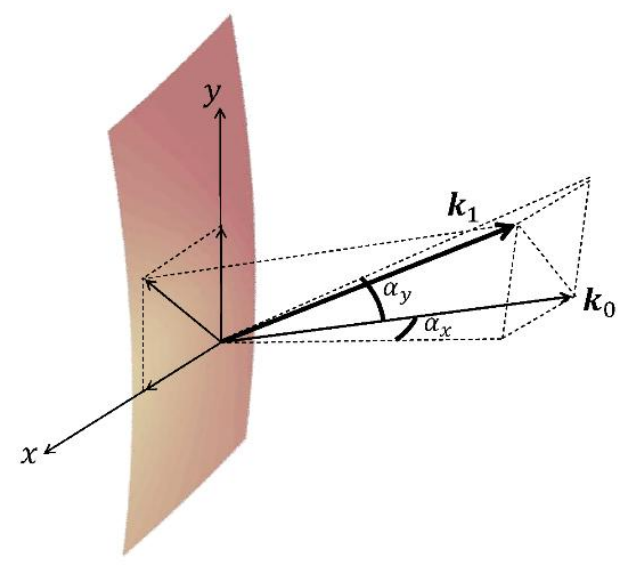

Fig. 3. (Color online) Schematic illustration of Angle of Arrival fluctuations on a given point in the wave-front.

\section{The Fried Parameter $\left(\mathbf{r}_{0}\right)$}

For a light beam propagating through the atmosphere, the Fried parameter $r_{0}$ is a length defined on the wave plane, in which the root mean square (rms) phase distortion over a circular area of diameter $\mathrm{r}_{0}$ is about one radian (Fried 1965; Roddier 1999).

\section{THEORETICAL CONSIDERATIONS}

Velocity, temperature and density fields are coupled to each other in fluid flows, and under turbulent conditions the scaling behavior of their fluctuations is an important observable (Landau and Lifshitz 1987; Frisch 1995). For an ideal gas, fluctuations in the density are linearly dependent on the refractive index $\mathrm{n}$, since from the Gladstone-Dale equation (Hartnett and Irvine 1970; Gladstone and Dale 1863)) we have:

$$
n=\rho \kappa+1,
$$

where $\kappa$ is the Gladstone-Dale constant for the gas. When a light beam passes through a turbulent medium, it is modified by correlated fluctuations in the refractive index scalar field, which are integrated over the propagation path. Indeed, it is possible to observe the scaling behavior which appears in the wave-front's phase distortions. We can define a second order phase structure function, $\mathrm{D}_{\varphi}(\mathrm{r})$, as

$D_{\varphi}(r)=\left\langle\left|\varphi\left(r^{\prime}\right)-\varphi\left(r^{\prime}+r\right)\right|^{2}\right\rangle$.

It has been shown (Fried 1966; Roddier 1999) that a Kolmogorov spectrum for refractive index fluctuations leads to a phase structure function given by

$D_{\varphi}(r)=6.88\left(r / r_{0}\right)^{5 / 3}$.

Taking into account the intermittent nature of turbulence requires that this be modified; in this case the phase structure function can then be generalized (Nicholls et al. 1995) as

$D_{\varphi}(r)=\gamma_{\beta}\left(r / r_{0}\right)^{\beta-2}$,

where $\beta$ is the exponent of the power spectrum for the index of refraction fluctuations (Silbaugh et al. 1996). The purpose of this Paper is to suggest a method of measuring $\beta$ using a new high-resolution instrument (Rasouli 2010).

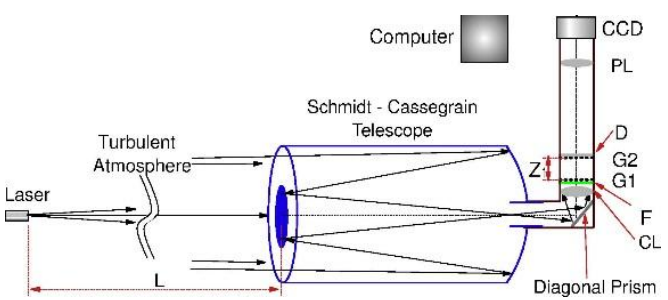

Fig. 4. (Color online) Schematic diagram of the experimental setup. CL, F, G1, G2, and PL, stand for the collimating lens, band pass filter, first grating, second grating, and the lens that projects the moiré pattern produced on the diffuser D on the CCD, respectively.

Experimentally, we pass a slightly divergent laser beam through the atmospheric boundary layer near ground level, which then enters into the telescope aperture (See Fig. 4). The laser beam is re-collimated behind the telescope's focal point and passes through a moiré deflectometer. As mentioned above, a fluctuating self-image of the first grating is formed on the second grating of the moiré deflectometer resulting in the formation of fluctuating moiré fringes which are then recorded on a CCD. Recording the successive moiré patterns by a CCD, after filtering the higher spatial frequencies, produces highly magnified AA fluctuations.

Covariance of the AA components are evaluated for pairs of points with a given horizontal separation on a single trace (normal to the grating rulings) or vertically between separate traces. By trace we refer to the determination of the minimum (maximum) value of intensity within the dark (bright) 
fringes. This can be done to one-pixel accuracy.

We choose a coordinate system such that the grating rulings are almost in the y-direction and the moire fringes in the $\mathrm{x}$-direction (See Fig. 5 for more details). In this arrangement the $\mathrm{x}$ component of the AA fluctuations can be measured, and longitudinal and transverse directions are referenced to the direction of the measured AA component. Two distinct structure functions for the AA fluctuations can be defined (Roddier 1981) from the relation

$D_{\alpha_{x}}(d)=\left\langle\left[\alpha_{x}(x, y)-\alpha_{x}(x+\mu, y+\eta)\right]^{2}\right\rangle$.

The longitudinal structure function $\mathrm{D}^{\mathrm{L}}{ }_{\alpha \mathrm{x}} \quad(\mathrm{d})$ and trans-verse structure function $\mathrm{D}^{\mathrm{T}}{ }_{\mathrm{ax}} \quad$ (d) correspond to $(\mu=d, \eta=0)$ and $(\mu=0, \eta=d)$, respectively.

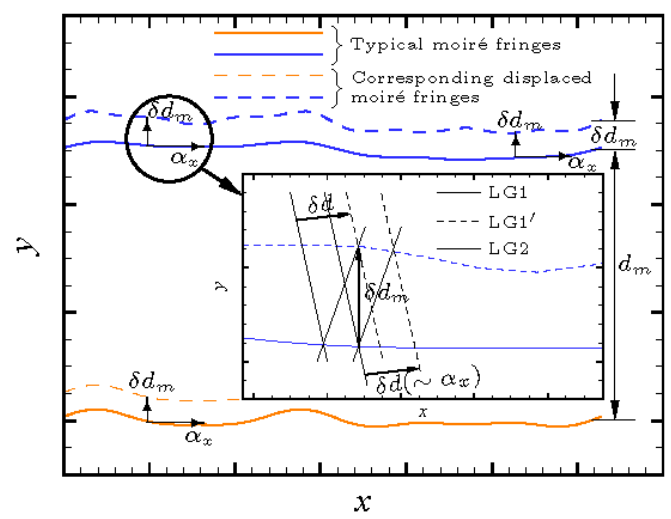

Fig. 5. (Color online) Illustration of longitudinal and transverse directions, longitudinal and transverse directions referred to the direction of the measured AA components. LG1, LG1', and LG2 are correspond to the typical line of the first grating, displaced first grating, and second grating, respectively. Here, longitudinal direction is parallel to the moiré fringe traces and transverse direction is perpendicular to the moiré fringe traces.

In this geometry, one can measure the $x$ component $\alpha_{\mathrm{x}}$, of the AA fluctuations (Rasouli 2010; Rasouli and Tavassoly 2006) by

$\alpha_{x}(x, y)=-\frac{\lambda}{2 \pi} \frac{\partial}{\partial x} \varphi(x, y)$,

where $\lambda$ and $\varphi$ are the wavelength and the wave-front phase, respectively. Hence, the covariance of the AA fluctuations

$$
B_{\alpha}(\mu, \eta)=\langle\alpha(x, y) \alpha(x+\mu, y+\eta)\rangle
$$

can easily be related to the structure function of phase $D_{\varphi}(\mu ; \eta)$ (Roddier 1999) by

$B_{\alpha}(\mu, \eta)=\frac{\lambda^{2}}{8 \pi^{2}} \frac{\partial^{2}}{\partial \mu^{2}} D_{\varphi}(\mu, \eta)$.

The generalized phase structure function of Eq. (4) for an anisotropic Kolmogorov model can be written as (Nicholls, Boreman, and Dainty 1995; Arad, Dhruva, Kurien, L'vov, Procaccia, and Sreenivasan 1998)
$D_{\varphi}(r)=\gamma_{\beta} r_{0}^{2-\beta}\left(\eta^{2}+\mu^{2}\right)^{(\beta-2) / 2}$.

One can obtain the following results for

longitudinal and transverse covariance (Nicholls, Boreman, and Dainty 1995)

$$
\begin{aligned}
& B_{\alpha}(d, 0)=0.0127 \gamma_{\beta} \lambda^{2} r_{0}^{2-\beta}(\beta-2)(b-3) d^{\beta-4}, \\
& B_{\alpha}(0, d)=0.0127 \gamma_{\beta} \lambda^{2} r_{0}^{2-\beta}(\beta-2) d^{\beta-4},
\end{aligned}
$$

respectively using the symbol $d$ in place of $\mu$ and $\eta$ for simplicity in the case where pure longitudinal and transverse components are considered. We note that a single value of $\beta$ has been used, valid for the case where the turbulence is roughly isotropic and homogeneous.

\section{EXPERIMENTAL RESULTS}

In Fig. 4, the schematic diagram of the experimental setup is shown (Rasouli 2010). For our measurements the second harmonic of a $\mathrm{CW}$ diode pumped Nd-YAG laser beam was passed through a turbulent surface layer of the atmosphere before entering the telescope (Meade 8 inch SchmidtCassegrain model) aperture. The light source and measurement set-up were installed at a height of 1 $\mathrm{m}$ over an asphalted area and separated by a distance $360 \mathrm{~m}$. The wavelength of the laser was $\lambda=532 \mathrm{~nm}$. A collimating lens CL with focal length $13.5 \mathrm{~cm}$ was used for collimation of the beam. Two gratings, G1 and G2, both with period $1 / 10 \mathrm{~mm}$ and dimension $20 \mathrm{~mm} \times 20 \mathrm{~mm}$, were installed at the ends of a cylindrical tube whose length could be adjusted in order to choose the desired Talbot distance. We have used $Z_{k}=1=3.7 \mathrm{~cm}$. In this setup, a projection lens $\mathrm{PL}$ with focal length $2.5 \mathrm{~cm}$ projects the image of the moiré patterns onto a CCD (model DMK 21AU), connected to a computer. The moiré fringes were recorded with a sampling rate of 60 frames/s. Several sets of experimental data were recorded and digitized; for all data, the wind moved in the same direction (specifically, from the west to the east) and its direction was nearly perpendicular to the beam propagation path.

Each data set was collected over an interval of $50 \mathrm{~s}$ and therefore contains 3000 frames. The typical recorded moiré pattern is shown in Fig. 6(a). In order to get clearer picture of the fringes, the images were enhanced using a spatial fast Fourier transform method to low pass filter the data and obtain Fig. 6(b). The displacement of either dark or bright traces in successive frames could be determined to within one-pixel accuracy. Typical traces of moiré fringe minima and maxima are shown in Fig. 6(b). Using the concept of virtual traces, the spatial resolution of the method in the direction perpendicular to the traces can be enhanced (Rasouli et al. 2010). Below, we refer to traces with intensities equal to the mean intensity of the adjacent bright and dark traces, as first order virtual traces (Rasouli et al. 2010; Dashti and Rasouli 2012).

Taking

$\alpha(x, y)=A s(x, y)$, 
the component $\alpha$ of the AA fluctuations is obtained. In Eq. (11) A is a constant coefficient and its value, $2.89 \times 10^{-6} \mathrm{rad} / \mathrm{pixel}$ is determined by $A=\left(f^{\prime} / f\right)\left(1 / Z_{k}\right)\left(d / d_{m}\right)$ where, $f^{\prime}, f, Z_{k}, d$, and $d_{m}$ are respectively the telescope focal length, the focal length of the collimating lens, the $\mathrm{k}^{\text {th }}$ Talbot distance of the first grating, the gratings' period, and the moire fringe spacing. $\mathrm{s}(\mathrm{x}, \mathrm{y})$ denotes the moiré fringe displacement corresponding to the point $(\mathrm{x}, \mathrm{y})$ on the aperture plane. For more details on the general method see Rasouli (2010).
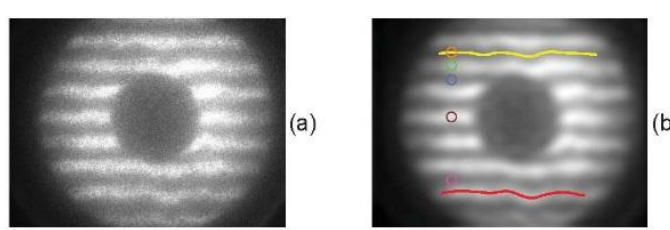

Fig. 6. (Color online) (a) Typical moiré pattern,

(b) the corresponding low frequency

illumination distribution. All of the moiré patterns consisted of $640 \times 480$ pixels. One pixel resolution in the image is equivalent to $0.35 \mathrm{~mm}$ in the real space on the telescope aperture plane. Yellow and red solid lines mark typical traces of moiré fringe minima and maxima, respectively. Time series of AA fluctuations in the positions of the color circles to be used in Fig. 7.

The plots in Fig. 7(a) to (e) show the horizontal component of AA fluctuations at four points on the telescope aperture versus time. These points are shown by the circles in Fig. 6(b). In these experiments, the digitized frames consisted of $640 \times 480$ pixels, $\mathrm{d}_{\mathrm{m}}$ was covered by 63 pixels, and the measurement precision of AA fluctuations was about 0.59 arc sec.

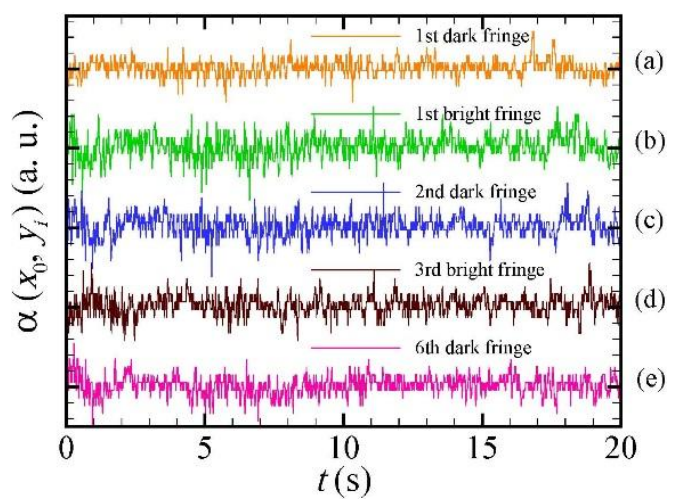

Fig. 7. (Color online) Typical horizontal components of AA fluctuations at four different points on the telescope aperture versus time. The location of the selected points are shown in Fig.

6. The data sets recorded in late evening in October.

From the fluctuations of the moiré fringe traces we calculate the longitudinal covariance of AA fluctuations, $B_{\alpha}(d, 0)$, for pairs of points displaced horizontally in space on a given trace. This process is performed for all traces corresponding to dark and bright fringes and repeated for all frames, and the mean value evaluated. In this calculation the minimum value of $\mathrm{d}$ corresponds to a length covered by a pixel and its maximum value corresponds to the maximum length of the traces. Similarly, the transverse covariance, $B_{\alpha}(0, d)$, is calculated for pairs of points displaced vertically in space on the telescope aperture plane (having the same $\mathrm{x}$ coordinate). Here, the minimum value of $\mathrm{d}$ corresponds to a distance of two adjacent dark and bright traces (i.e. $~ 31$ pixels) and its maximum value corresponds to the vertical distance between farthest two traces. By using two channel moiré deflectometers on a telescope, the spatial resolution of vertical displacements in $\mathrm{B}_{\alpha}(0, \mathrm{~d})$ can be improved to roughly the length covered by a single pixel, just as for the horizontal displacements in $\mathrm{B}_{\alpha}(\mathrm{d}, 0)$.

In Fig. 8, the calculated longitudinal and transverse covariances of $\mathrm{AA}$ fluctuations (i.e. $\mathrm{B}_{\alpha}(\mathrm{d}, 0)$ and $\left.B_{\alpha}(0, d)\right)$ for one particular data set are plotted.

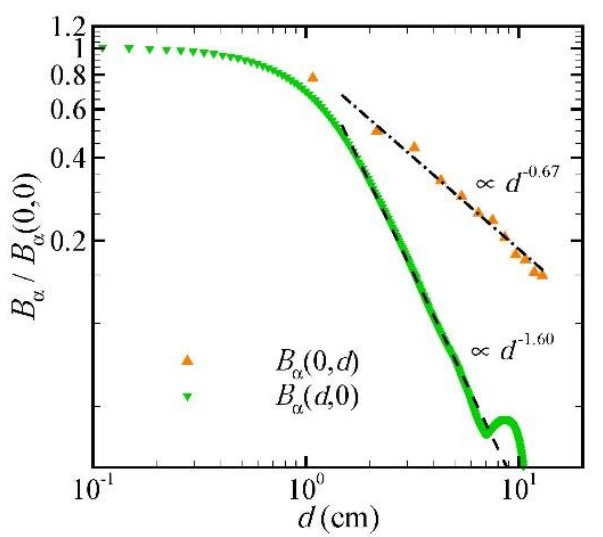

Fig. 8. (Color online) Calculated plots of the normalized longitudinal and transverse covariances of $\mathrm{AA}$ fluctuations (i.e. $B_{a}(d, 0)$ and $\left.B_{a}(0, d)\right)$ for data collected in late evening in October.

According to the Eqs. (9) and (10), a similar scaling form for the $\mathrm{B}_{\alpha}(\mathrm{d}, 0)$ and $\mathrm{B}_{\alpha}(0, \mathrm{~d})$ are expected. An important result from Fig. 8, is that the exponents of the longitudinal and transverse covariances of AA fluctuations differ significantly, demonstrating a strong anisotropy. A similar anisotropy was also observed for the velocity structure function at the atmospheric turbulence by Arad et al. (1998) using time series from a single hot wire pair.

Corresponding to the data shown in Fig. 8 we can identify two values of $\beta$ : $\beta_{1}=2.40$ and $\beta_{t}=3.33$. Here, $\beta_{1}$ and $\beta_{\mathrm{t}}$ are exponents corresponding to the longitudinal and transverse covariances of AA field, respectively. From Eq. (4) we would expect to find a value $\beta_{\mathrm{kol}} \sim 3.67$ for roughly isotropic and homogeneous turbulence, and indeed we observe something near to that but slightly closer to 3 for only one of the components. This, in fact, was also observed by Nicholls et al. (1995).

One can extend Eqs. (9) and (10) to include the observed anisotropy of the AA field

$B_{\alpha}(d, 0) \propto d^{\beta_{l}-4}$ 
$B_{\alpha}(0, d) \propto d^{\beta_{t}-4}$

respectively. It can be used for suggesting a modified formulation to include the anisotropy in the phase structure function.

Considering Fig. 8, we note that the scaling range starts at approximately $1 \mathrm{~cm}$ and ends at nearly 15 $\mathrm{cm}$. The latter is probably due to the telescope's aperture diameter.

\section{CONCLUSION}

In conclusion, we have applied a promising technique combining a moiré deflectometer with a telescope to observations of atmospheric turbulence near the ground surface. Specifically, we have calculated the exponents of the longitudinal and transverse covariances of AA fluctuations, which are measured by propagating a divergent laser beam through the atmospheric turbulence and into the aperture of a telescope forming the input of a moiré deflectometer. The observed anisotropy on the AA field can originate from two sources, strong temperature gradients and anisotropy in the velocity field near the surface, making correspondence to velocity structure functions challenging. Better characterization of the interplay between phase fluctuations and velocity and temperature fields will be the subject of a further study in controlled laboratory experiments, in which effects can be isolated, leading to proper deconvolution algorithms for field studies in addition to separate and precise measurements of the temperature field. The observed anisotropic features are indeed similar to those reported in the work of (Arad, Dhruva, Kurien, L'vov, Procaccia, and Sreenivasan 1998), albeit for the velocity increment. It is important to note that with this method we are able to directly measure the 2D spatial statistical properties of the AA field, due to the collection of large volume of 2D data across the telescope aperture, in contrast to the usual configuration of a moiré deflectometer which is sensitive to the incoming wave-front changes only in one direction normal to its rulings. As a final note we point out that this non-intrusive technique is also quite flexible: by choosing gratings of suitable pitch, size and displacement a wide range of turbulent conditions can be characterized with precision up to very high intensities. As a final note, we point out that the instrument, besides its other advantages, has the additional benefit of being relatively inexpensive.

\section{ACKNOWLEDGEMENTS}

S. Rasouli gratefully acknowledge support by the Institute for Advanced Studies in Basic Sciences (IASBS) Research Council under grant No. G2010IASBS107.

\section{REFERENCES}

Acton, D., R. Sharbaugh, J. Roehrig, and D. Tiszauer (1992). Wave-front tilt power spectral density from the image motion of solar pores. Appl. Opt. 31, 4280-4284.

Arad, I., B. Dhruva, S. Kurien, V. L'vov, I. Procaccia, and K. Sreenivasan (1998). Extraction of anisotropic contributions in turbulent flows. Phys. Rev. Lett. 81, 5330-5333.

Dashti, M. and S. Rasouli (2012). Measurement and statistical analysis of the wavefront distortions induced by atmospheric turbulence using twochannel moiré deflectometry. J. Opt. 14, 095704 .

Castaing, B., Y. Gagne, and E. Hopfinger (1990). Velocity probability density functions of high reynolds number turbulence. Physica D 46, 177.

Dayton, D., B. Pierson, B. Spielbusch, and J. Gonglewski (1992). Atmospheric structure function measurements with a shack-hartmann wave-front sensor. Opt. Lett. 17, 1737-1739.

Fried, D. (1965). Statistics of a geometrical representation of wave-front distortion. J. Opt. Soc. Am. 55, 1427-1435.

Fried, D. (1966). Optical resolution through a randomly inhomogeneous medium for very long and very short exposures. J. Opt. Soc. Am. 56, 1372.

Fried, D. (1975). Differential angle of arrival: theory, evaluation, and measurement feasibility. Radio Sci. 10, 71-76.

Frisch, U. (1995). Turbulence: the legacy of AN Kolmogorov. Cambridge: Cambridge University Press.

Gagne, Y., E. Hopfinger, and U. Frisch (1990). A new universal scaling for fully developed turbulence: the distribution of velocity increments in New Trends in Nonlinear Dynamics and Pattern-Forming Phenomena. New York: Plenum Press.

Gladstone, J. and T. Dale (1863). Researches or the refraction, dispersion, and sensitiveness of liquids. Phil. Trans. 153, 317.

Hartnett, J. and T. Irvine (1970). Advances in Heat Transfer. New York: Academic Press.

Hutchins, N., T. B. Nickels, I. Marusic, and M. Chong (2009). Hot-wire spatial resolution issues in wall-bounded turbulence. J. Fluid 
Mech. 635, 103-136.

Keren, E. and O. Kafri (1985). Diffraction effects in moiré deflectometry. J. Opt. Soc. Am. A 2, 111-120.

Landau, L. and E. Lifshitz (1987). Course of Theoretical Physics: Fluid mechanics, Translated by J. B. Sykes, W. H. Reid. Pergamon Press.

Lekakis, I. (1996). Calibration and signal interpretation for single and multiple hotwire/hot-film probes. Physica D 7, 1313.

Maurer, J., P. Tabeling, and G. Zocchi (1994). Statistics of turbulence between two counterrotating disks in low-temperature helium gas. Europhys. Lett. 26, 31.

Miles, R., W. Lempert, and B. Zhang (1991). Turbulent structure measurements by relief flow tagging. Fluid Dyn. Res. 8, 9.

Nicholls, T., G. Boreman, and J. Dainty (1995). Use of a shack-hartmann wavefront sensor to measure deviations from a kolmogorov phase spectrum. Opt. Lett. 20, 2460-2462.

Noullez, A., G. Wallace, W. Lempert, R. Miles, and U. Frisch (1997). Transverse velocity increments in turbulent flow using the relief technique. J. Fluid Mech. 339, 287.

Patorski, K. (1989). The self-imaging phenomenon and its applications. Progress in Optics 27, 1108.

Patorski, K. (1993). Handbook of the Moiré Fringe Technique. Elsevier.

Rasouli, S. (2010). Use of a moiré deflectometer on a telescope for atmospheric turbulence measurements. Opt. Lett. 35, 1470-1472.

Rasouli, S. (2012). Atmospheric Turbulence Characterization and Wavefront Sensing by Means of the Moiré Deflectometry", Topics in Adaptive Optics, Robert K. Tyson (Ed.). InTech.

Rasouli, S., M. Dashti, and A. N. Ramaprakash (2010). An adjustable, high sensitivity, wide dynamic range two channel wavefront sensor based on moiré deflectometry. Opt. Exp. 18, 23906.
Rasouli, S. and M. Tavassoly (2006). Application of moiré technique to the measurement of the atmospheric turbulence parameters related to the angle of arrival fluctuations. Opt. Lett. 31, 3276-3278.

Rasouli, S. and M. Tavassoly (2008). Application of the moiré deflectometry on divergent laser beam to the measurement of the angle of arrival fluctuations and the refractive index structure constant in the turbulent atmosphere. Opt. Lett. 33, 980-982.

Rayleigh, L. (1881). On copying diffractiongratings, and on some phenomena connected therewith. Philos. Mag. 11.

Roddier, F. (1981).The effects of atmospheric turbulence in optical astronomy. North-Holland: Academic Press.

Roddier, F. (1999). Adaptive optics in astronomy. Cambridge, United Kingdom: Cam-bridge university press.

Sarazin, M. and F. Roddier (1990). The ESO differential image motion monitor. Astron. Astrophys 227, 294.

Silbaugh, E., B. Welsh, and M. Roggemann (1996). Characterization of atmospheric turbulence phase statistics using wave-front slope measurements. J. Opt. Soc. Am. A 13, 24532460 .

Talbot, H. (1836). LXXVI. Facts relating to optical science. No. IV. Philos. Mag. 9, 401-407.

Volk, R., E. Calzavarini, G. Verhille, D. Lohse, N. Mordant, J. Pinton, and F. Toschi (2008). Acceleration of heavy and light particles in turbulence: Comparison between experiments and direct numerical simulations. Physica $D$ 237, 2084-2089.

Volk, R., N. Mordant, G. Verhille, and J. Pinton (2008). Laser Doppler measurement of inertial particle and bubble accelerations in turbulence. EPL (Europhysics Letters) 81, 34002 .

Webster, M. (2009). Simplification of RELIEF Velocimetry Using Pico-Second Tagging and Broad Band Interrogation. Ph. D. thesis, The Ohio State University. 